\title{
Clinicopathologic and molecular analysis of disseminated appendiceal mucinous neoplasms: identification of factors predicting survival and proposed criteria for a three-tiered assessment of tumor grade
}

Jon M Davison ${ }^{1}$, Haroon A Choudry ${ }^{2}$, James F Pingpank ${ }^{2}$, Steven A Ahrendt ${ }^{2}$, Matthew P Holtzman ${ }^{2}$, Amer H Zureikat ${ }^{2}$, Herbert J Zeh², Lekshmi Ramalingam², Benjamin Zhu ${ }^{1}$, Marina Nikiforova ${ }^{1}$, David L Bartlett ${ }^{2}$ and Reetesh K Pai ${ }^{1}$

${ }^{1}$ Department of Pathology, University of Pittsburgh Medical Center, Pittsburgh, PA, USA and ${ }^{2}$ Department of Surgery, Division of Surgical Oncology, University of Pittsburgh Medical Center, Pittsburgh, PA, USA

Previous studies have demonstrated that the prognosis of disseminated mucinous appendiceal neoplasms is highly dependent upon tumor grade. Reflecting this, the 7th edition of the American Joint Committee on Cancer (AJCC) staging system now incorporates a three-tier grading system for prognostic staging of mucinous appendiceal tumors. However, the grading criteria are not well described. In order to address this issue, we evaluated clinicopathologic and molecular features of 219 cases from 151 patients with widely disseminated appendiceal mucinous neoplasia treated at our institution between 2004 and 2012. We identified histologic features that were associated with worse overall survival on univariate analysis: destructive invasion, high cytologic grade, high tumor cellularity, angiolymphatic invasion, perineural invasion, and signet ring cell component (all with $\boldsymbol{P}<\mathbf{0 . 0 0 0 1}$ ). We used these morphologic characteristics to classify neoplasms into three grades: AJCC grade G1 lacked all adverse histologic features; AJCC grade G2 had at least one adverse histologic feature (except a signet ring cell component); and AJCC grade G3 were defined by the presence of a signet ring cell component. Patients with AJCC grade G2 and grade G3 adenocarcinomas had a significantly worse prognosis compared with AJCC grade G1 $(P<\mathbf{0 . 0 0 0 1}$ for each). A trend toward worse overall survival was identified for patients with AJCC grade G3 adenocarcinomas compared with AJCC grade G2 adenocarcinomas $(P=0.07)$. Our multivariate analysis found that this three-tier grading system was a significant predictor of outcome $(P=\mathbf{0 . 0 0 8})$, independent of other prognostic variables. After controlling for other prognostic variables, AJCC grade G2 was associated with a 2.7-fold increased risk of death (95\% confidence interval (CI), 1.2-6.2) and AJCC grade G3 was associated with a 5.1-fold increased risk of death (95\% $\mathrm{Cl}, 1.7-14)$ relative to grade $\mathrm{G} 1$ tumors. Our results indicate that evaluation of a limited set of adverse histologic features allows for the separation of disseminated mucinous neoplasms of appendiceal origin into three morphologically defined and prognostically relevant grades as advocated by the AJCC.

Modern Pathology (2014) 27, 1521-1539; doi:10.1038/modpathol.2014.37; published online 14 March 2014

Keywords: appendix; disseminated peritoneal adenomucinosis; low-grade mucinous neoplasm; mucinous adenocarcinoma; peritoneal mucinous carcinomatosis; pseudomyxoma peritonei

Correspondence: Dr RK Pai, MD, University of Pittsburgh, Department of Pathology, Presbyterian Hospital, 200 Lothrop Street, Room A-610, Pittsburgh, PA 15213, USA.

E-mail: pair@upmc.edu

Received 14 October 2013; accepted 14 January 2014; published online 14 March 2014
Despite advances in our understanding of appendiceal mucinous neoplasms and their relationship to the pseudomyxoma peritonei syndrome, the classification of mucinous tumors of the appendix is still confusing. Most cases of classic pseudomyxoma peritonei, a clinical entity characterized by grossly 
evident, diffuse, intra-abdominal mucinous ascites involving the peritoneal surfaces, develop as a result of a mucinous neoplasm arising in the appendix. ${ }^{1-5}$ Several studies have demonstrated that disseminated appendiceal mucinous neoplasms exhibit a wide spectrum of clinical behavior, ranging from neoplasms which are relatively slow-growing but with considerable risk for recurrence and eventual death and those neoplasms that are highly aggressive with increased likelihood of early death. ${ }^{6-11}$

There are multiple clinical factors that influence survival in patients with disseminated mucinous appendiceal neoplasms, but pathologic grade has repeatedly been shown to be an independent prognostic factor. ${ }^{6-9,11,12}$ Proposed terminology for lowgrade tumors has included disseminated peritoneal adenomucinosis, ${ }^{11}$ low-grade mucinous carcinoma peritonei, ${ }^{7}$ low-grade mucinous neoplasm, ${ }^{8,9}$ and well-differentiated mucinous adenocarcinoma. ${ }^{13}$ Terminology proposed for high-grade tumors has included peritoneal mucinous carcinomatosis, ${ }^{11}$ high-grade mucinous adenocarcinoma, ${ }^{14}$ and highgrade mucinous carcinoma peritonei. ${ }^{7}$ Given the divergent treatment approaches that may be used, it is imperative that the diagnostic pathology report attempt to unambiguously differentiate mucinous neoplasms that are likely to behave in an indolent fashion from those that are likely to behave aggressively. For patients with clinically indolent, low-grade disease, cytoreductive surgery with a goal of dramatically reducing or eliminating all tumor burden has been shown to substantially prolong survival. ${ }^{15-20}$ Systemic chemotherapy is believed to be of little benefit for most patients with indolent peritoneal disease. ${ }^{12,15,21}$ For patients with disseminated high-grade mucinous adenocarcinoma that is likely to pursue a more aggressive clinical course, the inherent risks of aggressive surgical debulking and cytoreduction must be carefully considered against the reduced likelihood of prolonged survival. Although its effectiveness is not well established, systemic chemotherapy is more likely to be incorporated into a multimodal treatment strategy for tumors that are judged to be clinically aggressive. ${ }^{15,22,23}$

In an attempt to codify the diagnostic terminology of appendiceal mucinous neoplasms, both the 4th edition of the World Health Organization (WHO) Classification of Tumors of the Digestive System and the 7th edition of the American Joint Committee on Cancer (AJCC) Staging Manual now differentiate low-grade mucinous neoplasms from high-grade, but they differ in their terminology and grading schemes. ${ }^{24,25}$ The WHO identifies morphologic characteristics (architecture, cytology, presence of signet ring cells and mitotic activity) that can be used to classify low- and high-grade tumors. ${ }^{6}$ The three-tiered approach adopted by the AJCC (lowgrade tumors are classified as grade G1, but highgrade tumors may be classified as grade G2 or grade
G3) would appear to offer a necessary refinement. Unfortunately, the AJCC did not provide any criteria that should be used to classify tumor grade nor were any studies cited that could be used as a guide for tumor grading. The only citation, Bradley et al., ${ }^{7}$ concluded that a two-tiered system was preferable to a three-tiered system. The AJCC system also uses the descriptive terminology well-, moderately and poorly differentiated in parallel with the alphanumeric grades (G1, G2, and G3, respectively). These descriptive terms that are widely used to grade other gastrointestinal cancers seem in our experience to be particularly confusing and difficult to apply directly to mucinous tumors of the appendix. Despite the convergence of the two widely accepted classification systems of the WHO and AJCC around the idea that disseminated appendiceal mucinous tumors should be graded in order to predict clinical behavior, we feel that the lack of explicit criteria and dissimilarity in the grading schemas engenders considerable variability among pathologists over the classification of appendiceal mucinous neoplasms. In our own institution, we found that this inconsistency translated into diagnostic pathology reports that fail to covey clinically relevant information and led to considerable confusion among treating physicians.

To evaluate the utility of a criteria-based, threetier grading system as proposed by the AJCC, we have reviewed the clinicopathologic and molecular features of 219 mucinous appendiceal neoplasms from 151 patients with pseudomyxoma peritonei treated over an 8-year period at our institution. We developed criteria for AJCC grade G1, grade G2 and grade G3 disseminated mucinous appendiceal tumors that are based on morphological features and demonstrated the prognostic utility of this classification system for predicting overall survival while controlling for other prognostic variables. Last, we evaluated the complementary prognostic utility of molecular testing for loss of heterozygosity (LOH) in the context of the AJCC-based three-tier grading system.

\section{Materials and methods}

\section{Study Group}

The clinicopathologic records of 151 patients with primary mucinous neoplasms of the appendix accessioned at the Department of Pathology, University of Pittsburgh Medical Center for the years 2004-2012 were reviewed. The pathology reports and hospital charts were reviewed and the following information was obtained: type of initial surgical procedure, use of hyperthermic intraperitoneal chemotherapy at the time of surgery, the presence or absence of residual macroscopic intraperitoneal disease after initial surgical debulking procedure, and the initial pathologic diagnosis from the original 
pathology report. Demographic, intraoperative, and clinical follow-up data were obtained from hospital and clinic charts under the guidelines of the University of Pittsburgh Institutional Review Board (IRB\# PR012020335).

\section{Pathologic Evaluation}

All slides from the original surgery and subsequent procedures were reviewed. The following histologic features were recorded for each case: cytologic grade, tumor cellularity, destructive invasion, histologic pattern of destructive invasion, signet ring cell component, lymph node involvement, angiolymphatic invasion, and perineural invasion. The primary appendiceal neoplasm, when available, was also evaluated for these histologic features. The pathology review was conducted in a blinded fashion to the clinical follow-up data.

Cytologic grade was scored as low or high grade, as previously described. ${ }^{8}$ Low grade was defined by the presence of flat strips of cells with mildly enlarged, hyperchromatic nuclei with nuclear stratification and maintenance of cell polarity without significant mitotic activity or prominent nucleoli. High grade was defined by the presence of any enlarged, vesicular nuclei with full-thickness stratification, loss of nuclear polarity, prominent nucleoli, cribriform or micropapillary growth, and increased mitotic figures, which often extended to the luminal aspect of the epithelial cell. Any tumor with signet ring cells was defined as high cytologic grade. For the purpose of subgroup analysis, we separately identified and recorded a minor subset of neoplasms with predominantly low cytologic grade that had focal areas $(<10 \%$ of the total tumor) with noticeable nuclear stratification and mild to moderate nuclear enlargement that was insufficient for the designation of high cytologic grade. We provisionally classified this as 'increased proliferation' in otherwise low cytologic grade tumors.

Overall tumor cellularity was divided into three groups: acellular, low cellularity, and high cellularity. Each tumor was categorized into one of these groups based on the overall percent of tumor that contained neoplastic epithelium within mucinous deposits by visual estimation of the entire case at low-power magnification $(\times 20)$. Tumors labeled as acellular were composed entirely of mucin without identifiable neoplastic mucinous epithelium after extensive histologic sampling. Tumors labeled as low cellularity had neoplastic epithelium present within mucin which accounted for $<20 \%$ of the mucinous component. Tumors labeled as high cellularity had neoplastic epithelium present within mucin which accounted for $\geq 20 \%$ of the mucinous pools.

To qualify as invasion, we required the presence of unequivocal infiltration into subjacent normal tissues with the following histologic patterns: (1) infiltrating, haphazard, irregular, jagged neoplastic glands or single cells associated with desmoplastic stromal reaction, (2) expansile and confluent cribriform glandular growth, or (3) small nests, glands, or single neoplastic cells floating within small pools of mucin with or without desmoplastic stromal reaction. When present, the predominant histologic pattern of destructive invasion was recorded for each case. Neoplasms that predominantly lacked destructive invasion but demonstrated questionable and focal areas of invasion involving at most a single low-power $(\times 40)$ field were separately recorded and provisionally designated as having increased proliferation.' Importantly, the presence of neoplastic mucinous epithelium and mucin within the wall of intestinal organs or involving the spleen, ovaries, omentum, and liver parenchyma was not sufficient for a diagnosis of invasion. Appendical mucinous neoplasms frequently involve these organs and display a 'pushing' border without unequivocal, haphazard infiltration.

Signet ring cells were defined as infiltrating tumor cells with prominent intracytoplasmic mucin displacing and indenting the nucleus. Isolated degenerating mucinous neoplastic epithelial cells floating within mucin pools with signet ring cell-like morphology were not considered to represent signet ring cells. When present, the percentage of tumor composed of signet ring cells were recorded as $1-10 \%, 10-50 \%,>50-95 \%$, and $>95 \%$.

\section{Molecular Analysis}

KRAS mutational analysis. Tumor targets were manually microdissected from $4-\mu \mathrm{m}$ unstained histologic sections using the original hematoxylin and eosin-stained slide as a guide. DNA was isolated from each target using the DNeasy kit (Qiagen, Valencia, CA, USA), according to the manufacturer's instructions. DNA was amplified with primers flanking exon 2 of the KRAS gene (forward primer 5'-GGT GAG TTT GTA TTA AAA GGT ACT GG-3' and reverse primer $5^{\prime}$-TCC TGC ACC AGT AAT ATG CA-3'). PCR products were sequenced in both sense and antisense directions using the BigDye Terminator v3.1 cycle sequencing kit on an ABI 3130 (Applied Biosystems Inc., Foster City, CA, USA), according to the manufacturer's instructions. The sequences were analyzed using Mutation Surveyor software (SoftGenetics, LLC, State College, PA, USA). Each case was classified as positive or negative for KRAS mutation based on the sequencing results.

LOH analysis. Tumor and normal tissue targets were manually microdissected from $4-\mu$ m unstained histologic sections using the original hematoxylin and eosin-stained slide as a guide. DNA was isolated from each target using the DNeasy kit (Qiagen), according to the manufacturer's instructions. Each 
sample was analyzed by 16 PCR reactions for individual polymorphic microsatellites situated at nine genomic regions $(1 p, 3 p, 5 q, 7 q, 9 p, 9 q, 10 q$, $17 p$, and 18q) in proximity to tumor suppressor genes which have previously found to be mutated in patients with disseminated appendiceal mucinous neoplasms, as previously described. ${ }^{26}$ The Genbank accession information for the microsatellite markers and cytogenetic localization are as follows: D1S407: 1p36.21; D1S171: 1p36; D3S1539: 3p26; D3S516: 3p26; D5S1384: 5q23; D5S615: 5q23; D7S486: 7q31; D7S1530: 7q31; D9S251: 9p21; D9S1748: 9p21; D9S252: 9q24; D10S520: 10q23; D10S1173: 10q23; D17S974: 17p13; D17S1289: 17p13; D18S487: 18q21. The PCR products were analyzed by capillary electrophoresis on an ABI 3130 (Applied Biosystems). Normal tissue was examined to determine whether the patient is heterozygous for a particular marker (genetically informative) and neoplastic tissue was analyzed to detect the LOH. Allele peak heights and lengths were used to define the presence or absence of allelic loss imbalance, as previously described. ${ }^{26}$ The fractional mutation rate was calculated for each sample and defined as the number of mutated markers divided by the total number of informative markers and expressed as a percent. A fractional mutation rate $<25 \%$ was defined as low fractional mutation rate, whereas a fractional mutation rate $\geq 25 \%$ was defined as high fractional mutation rate. $^{26}$

\section{Statistical Analysis}

Chi-square or Fisher exact tests were used to characterize the relationship between categorical variables, as appropriate. The inter-observer reproducibility of the overall grade assessment was assessed using the kappa statistic. The end points selected for survival analysis included overall survival and time to disease progression. Overall survival was defined as the time (measured in months) from the date of initial diagnosis to the date of death and censored at the date of last clinical follow-up. Time to disease progression was defined as time (measured in months) from initial therapy to the first documented clinical evidence of progression or death and censored at the time of last clinical contact or death. Survival rates were determined by the Kaplan-Meier method and differences between groups were evaluated by log-rank test. Hazard ratios were calculated from a Cox proportional hazard model to identify individual predictors of overall survival. Multivariate analysis of significant or borderline individual risk factors $(P<0.1)$ was performed using Cox proportional hazard regression to identify independent risk factors for overall survival. Statistical analyses were performed using SPSS (for Windows 12.0, SPSS Inc., Chicago, IL, USA).

\section{Results}

\section{Pathologic Findings in Disseminated Mucinous Appendiceal Tumors}

Identification of histologic risk factors associated with poor overall survival. A total of 219 cases from 151 patients were reviewed with a median number of 27 slides per case (range 2-77 slides). All tumors included in the study had widespread peritoneal disease beyond the right lower quadrant, hence, extent of disease could not be scored as a risk factor for survival. Tumors were classified according to the histopathologic features assessed at the time of the initial tumor debulking procedure.

The two most common adverse histologic features in the cohort of 151 patients were destructive invasion (present in 54\% of tumors) and high cytologic grade (present in 50\% of tumors). High tumor cellularity was seen in $44 \%$, signet ring cells in $18 \%$, angiolymphatic invasion in $18 \%$, and perineural invasion in $15 \%$. Sixty-nine cases $(46 \%)$ had none of these unfavorable histologic features, $48(32 \%)$ had 1-3 unfavorable characteristics; and $34(23 \%)$ cases had more than three unfavorable histologic characteristics.

As expected, we found in our univariate analysis that there was a significant adverse association between all of the individual unfavorable pathologic characteristics and overall survival (Table 1). These

Table 1 Histologic features used to grade disseminated mucinous neoplasms are adverse risk factors in univariate analysis of overall survival

\begin{tabular}{|c|c|c|c|}
\hline Histologic feature & $\begin{array}{c}\text { No. of } \\
\text { patients }^{\mathrm{a}}\end{array}$ & $\begin{array}{l}\text { Hazard ratio } \\
(95 \% \text { CI) }\end{array}$ & P-value \\
\hline \multicolumn{4}{|l|}{ Cytologic grade } \\
\hline Low $^{b}$ & 71 & Referent & \multirow[t]{2}{*}{$<0.001$} \\
\hline High & 70 & $4.61(2.46-8.66)$ & \\
\hline \multicolumn{4}{|l|}{ Cellularity } \\
\hline Low $(<20 \%)^{b}$ & 80 & Referent & \multirow[t]{2}{*}{$<0.001$} \\
\hline $\operatorname{High}(>20 \%)$ & 61 & $2.83(1.63-4.94)$ & \\
\hline \multicolumn{4}{|c|}{ Destructive invasion } \\
\hline Absent & 66 & Referent & \multirow{2}{*}{$<0.001$} \\
\hline Present & 75 & $5.59(2.80-11.14)$ & \\
\hline \multicolumn{4}{|l|}{ Signet ring cells } \\
\hline Absent & 114 & Referent & \multirow[t]{2}{*}{$<0.001$} \\
\hline Present & 27 & $3.45(1.9-6.3)$ & \\
\hline \multicolumn{4}{|c|}{ Angiolymphatic invasion } \\
\hline Absent & 116 & Referent & \multirow[t]{2}{*}{$<0.001$} \\
\hline Present & 25 & $2.91(1.61-5.25)$ & \\
\hline \multicolumn{4}{|l|}{ Perineural invasion } \\
\hline Absent & 120 & Referent & \multirow[t]{2}{*}{$<0.001$} \\
\hline Present & 21 & $4.36(2.41-7.89)$ & \\
\hline
\end{tabular}

Abbreviation: CI, confidence interval.

${ }^{a}$ Ten patients had no clinical follow-up and were excluded from this analysis.

${ }^{\mathrm{b}}$ Cases with mucin only (acellular) are included in the low cytologic grade and low-cellularity category for the univariate analysis. 
histologic features (high cytologic grade, high cellularity, destructive invasion, angiolymphatic invasion, perineural invasion, and signet ring cells) were used to classify all cases into three histologic grades as adopted by the AJCC. We defined tumors that lacked all adverse pathologic risk factors as low grade (AJCC grade G1). The presence of signet ring cell adenocarcinoma was considered high grade (AJCC grade G3). Tumors with any number of other adverse pathologic risk factors (excluding signet ring cells) were defined as high grade (AJCC grade G2).

Inter-observer reproducibility of overall AJCC grade assessment. All 219 cases from 151 patients were assigned an overall AJCC grade by one pathologist (RKP). For the purposes of evaluating interobserver reproducibility in overall AJCC grade assessment, one additional pathologist (JMD) evaluated overall AJCC grade for a consecutive series of pathologic slides from the initial tumor debulking procedure from 114 patients. There was exact agreement in tumor grade assessment (G1, G2, and G3) in 107/114 cases reviewed by two pathologists representing very strong inter-observer concordance $(\kappa=0.906$, percent concordance 94\%). All seven initially discordant grade assessments were reviewed (RKP and JMD) to establish a consensus AJCC grade.

Differentiating between AJCC grade G2 and AJCC grade G3 adenocarcinoma accounted for 4/7 discordant cases. In all four cases, the presence of focal areas of mucinous neoplastic epithelial cells with signet ring cell-like morphology resulted in discordant grade assessment. For three cases, the tumor cells with signet ring cell-like morphology comprised $<5 \%$ of the overall tumor. These areas were considered to represent isolated degenerating mucinous neoplastic epithelial cells floating within mucin pools within an otherwise AJCC grade G2 adenocarcinoma. For one case, the tumor cells with signet ring cell morphology accounted for $10-20 \%$ of the overall tumor and demonstrated infiltration into subjacent stroma; this tumor was classified as AJCC grade G3 adenocarcinoma.

Differentiating between AJCC grade G1 and AJCC grade G2 adenocarcinoma accounted for 3/7 discordant cases. For all three cases, there was disagreement in the assessment of invasion. For two cases, a focal area of questionable invasion involving a single low-power $(\times 40)$ field was identified; these two cases were classified as AJCC grade G1 neoplasms with increased proliferation.

Pathologic findings in disseminated low-grade mucinous neoplasm (AJCC Grade G1). A total of 69 patients had low-grade mucinous neoplasms (AJCC grade G1) at the time of initial surgery (Table 2) with 27 patients having subsequent tumor resections available for review following the initial surgical debulking attempt. These tumors were characterized by a predominance of copious extracellular mucin within the peritoneal cavity (Figure 1a). Neoplastic mucinous epithelium accounted for $<20 \%$ of the mucinous component of the tumor. Most tumors (64/69, 93\%) demonstrated strips and clusters of mucinous epithelium with low cytologic grade within large mucin pools (Figure 1b). A small subset of cases $(5 / 69,7 \%)$ were composed entirely of acellular mucinous peritoneal deposits after extensive histologic evaluation for a cellular component.

Lymph node involvement was very rare in lowgrade tumors in comparison with high-grade tumors (Table 2). A single case $(1 / 69,1 \%)$ demonstrated involvement of a single lymph node. By definition, the tumor lacked any other adverse histologic features. The patient had history of recent abdominal surgery and the lymph node involvement was thought to represent tumor dissection into a lymph node likely related to the patient's previous surgery rather than a lymphatic metastasis.

Eight of 69 tumors (12\%) were predominantly low grade but demonstrated focal areas with increased nuclear atypia or a questionable microscopic focus of invasion and were designated as having 'increased proliferation.' There were three tumors with predominantly low cytologic grade but they had focal areas with increased cytologic atypia including mild nuclear enlargement and nuclear stratification, insufficient for a diagnosis of high cytologic grade (Figure 1c). In five cases, there was a focal microscopic area exhibiting small mucin pools with floating clusters of neoplastic mucinous epithelium with low cytologic grade that raised concern for, but was not diagnostic of, destructive stromal invasion (Figures 1e and f). In three cases, the areas concerning for invasion were within the small bowel muscularis propria. In the remaining two cases, these foci were within the omentum.

The primary appendix was available for review in 34 of 69 patients $(49 \%)$ with disseminated AJCC grade G1 neoplasms. In 32 of 34 cases, the primary appendix lacked all adverse histologic features. However, in two cases, the primary neoplasm within the appendix demonstrated focal areas with sufficient nuclear features to warrant the designation of high cytologic grade; however, the primary neoplasm did not demonstrate any additional adverse histologic features. In both of these cases, the peritoneal disease was composed exclusively of low cytologic grade and low-cellularity disease without any adverse histologic features.

Pathologic findings in disseminated high-grade mucinous adenocarcinoma (AJCC Grade G2). A total of 55 patients comprise this group (Table 2) with 18 patients having subsequent tumor resections available for review following the initial surgical debulking attempt. By definition, highgrade mucinous adenocarcinomas (AJCC grade G2) demonstrated at least one adverse histologic feature but lacked a signet ring cell component. The tumors from these 55 patients demonstrated the following 
Table 2 Clinicopathologic features stratified by grade

\begin{tabular}{|c|c|c|c|c|c|}
\hline Clinical and pathologic features & $\begin{array}{c}\text { Low-grade } \\
\text { (AJCC Grade G1) } \\
\mathrm{N}(\%)\end{array}$ & $\begin{array}{c}\text { High-grade } \\
\text { (AJCC Grade G2) } \\
\text { N (\%) }\end{array}$ & $\begin{array}{c}\text { High-grade } \\
\text { (AJCC Grade G3) } \\
\text { N (\%) }\end{array}$ & P-value & P-value $\mathrm{b}$ \\
\hline No. of patients & 69 (100) & $55(100)$ & $27(100)$ & NA & NA \\
\hline Sex, male/female & $34(49) / 35(51)$ & $30(55) / 25(45)$ & $13(48) / 14(52)$ & 0.800 & 0.643 \\
\hline Mean age in years (range) & $53(24-84)$ & $53(32-72)$ & $54(31-70)$ & 0.280 & 0.299 \\
\hline \multicolumn{6}{|c|}{ Macroscopic residual disease after initial surgical debulking } \\
\hline Not assessed & 5 & 2 & 2 & 0.200 & 0.810 \\
\hline Absent (R0) & $34(53)$ & $26(49)$ & $13(52)$ & & \\
\hline Present (R1) & $30(47)$ & $27(51)$ & $12(48)$ & & \\
\hline \multicolumn{6}{|l|}{ Intraperitoneal chemotherapy } \\
\hline HIPEC given & $61(88)$ & $42(76)$ & $21(77)$ & 0.179 & 1.000 \\
\hline No HIPEC given & $8(12)$ & $13(24)$ & $6(23)$ & & \\
\hline \multicolumn{6}{|l|}{ Lymph node involvement } \\
\hline Absent & $68(99)$ & $45(83)$ & $7(28)$ & $<0.001$ & $<0.001$ \\
\hline Present & $1(1)$ & $9(17)$ & $18(72)$ & & \\
\hline \multicolumn{6}{|l|}{ Cytologic grade } \\
\hline Low grade & $69(100)$ & $7(13)$ & 0 & $<0.001$ & 0.089 \\
\hline High grade & 0 & $48(87)$ & $27(100)$ & & \\
\hline \multicolumn{6}{|l|}{ Cellularity } \\
\hline None (mucin only) & $5(7)$ & 0 & 0 & $<0.001$ & 0.075 \\
\hline Low $(<20 \%)$ & $64(93)$ & $14(25)$ & $2(7)$ & & \\
\hline High $(\geq 20 \%)$ & 0 & $41(75)$ & $25(93)$ & & \\
\hline \multicolumn{6}{|l|}{ Destructive invasion } \\
\hline Absent & $69(100)$ & $1(2)$ & 0 & $<0.001$ & 1.000 \\
\hline Present & 0 & $54(98)$ & $27(100)$ & NA & NA \\
\hline \multicolumn{6}{|l|}{ Pattern of destructive invasion } \\
\hline Small mucin pools & 0 & $33(60)$ & $7(26)$ & & \\
\hline Complex architecture & 0 & $6(11)$ & 0 & & \\
\hline Tubules, single cells & 0 & $15(27)$ & $20(74)$ & & \\
\hline \multicolumn{6}{|l|}{ Signet ring cells } \\
\hline Absent & $69(100)$ & $55(100)$ & 0 & NA & NA \\
\hline Present & 0 & 0 & $27(100)$ & & \\
\hline \multicolumn{6}{|l|}{ Angiolymphatic invasion } \\
\hline Absent & $69(100)$ & $47(85)$ & $8(30)$ & $<0.001$ & $<0.001$ \\
\hline Present & 0 & $8(15)$ & $19(70)$ & & \\
\hline \multicolumn{6}{|l|}{ Perineural invasion } \\
\hline Absent & $69(100)$ & $52(95)$ & $8(30)$ & $<0.001$ & $<0.001$ \\
\hline Present & 0 & $3(5)$ & $19(70)$ & & \\
\hline \multicolumn{6}{|l|}{ Total number of adverse features ${ }^{C}$} \\
\hline 0 & $69(100)$ & 0 & 0 & $<0.001$ & $<0.001$ \\
\hline $1-3$ & 0 & $47(86)$ & $1(4)$ & & \\
\hline$>3$ & 0 & $8(14)$ & $26(96)$ & & \\
\hline
\end{tabular}

Abbreviations: AJCC, American Joint Committee on Cancer; FMR, fractional mutation rate; HIPEC, hyperthermic intraperitoneal chemotherapy. ${ }^{\text {a }} P$-value for comparison across all groups.

${ }^{\mathrm{b}} P$-value for the comparison of grade G2 and grade G3.

${ }^{\mathrm{C}}$ Includes: high cytologic grade, high cellularity, destructive invasion, signet ring cells, angiolymphatic invasion, and perineural invasion.

number of adverse histologic features: 1 adverse feature, 4 patients $(7 \%) ; 2$ adverse features, 12 patients (22\%); 3 adverse features, 31 patients (56\%); 4 adverse features, 7 patients (13\%); and 5 adverse features, 1 patient (2\%). The most frequent adverse histologic features observed in the initial surgical specimens from the 55 patients were destructive invasion (98\%), high cytologic grade (87\%), and high tumor cellularity (75\%) (Figures 2 and 3). When present, destructive invasion and high tumor cellularity were identified in the majority of the tumor. However, with respect to cytologic grade, some neoplasms were heterogeneous showing areas with low cytologic grade admixed with areas 


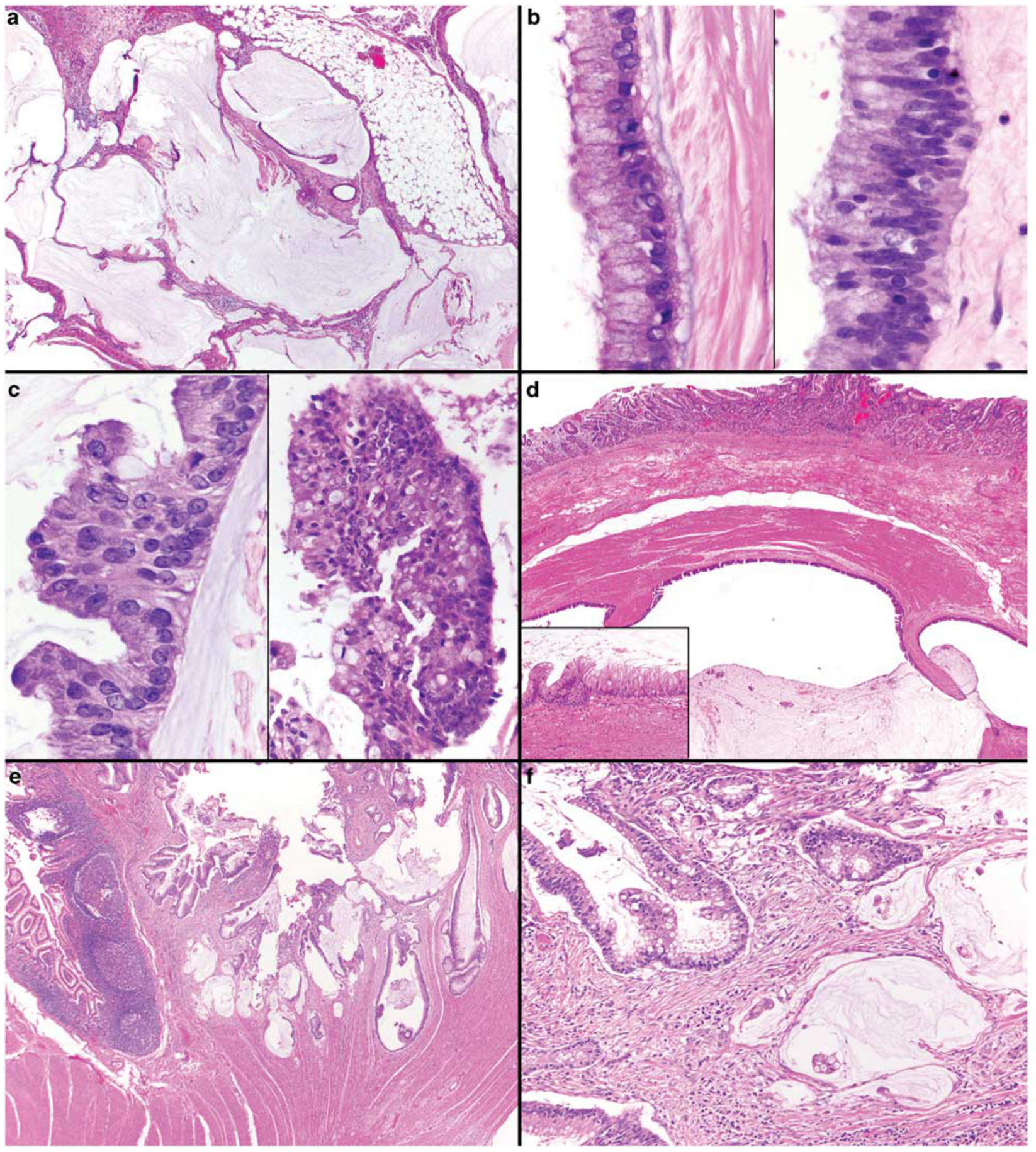

Figure 1 (a) Disseminated low-grade appendiceal mucinous neoplasm (AJCC grade G1) characterized by copious mucin pools with scattered strips of mucinous epithelium accounting for $<20 \%$ of tumor volume $(\times 20)$. (b) A spectrum of nuclear cytologic changes was characterized as low-grade, including basally located small round nuclei (left half) and mildly stratified oval-shaped nuclei (right half) $(\times 400)$. (c) A subset of cases demonstrated focal areas with increased cytologic atypia including mild nuclear enlargement and nuclear stratification but without unequivocal high-grade nuclear cytologic features (left panel $\times 400$, right panel, $\times 200$ ). Importantly in this subset of cases, these areas accounted for $<10 \%$ of the tumor with the remainder of the tumor composed of neoplastic epithelium with low cytologic grade. In our series, these tumors were labeled as predominantly low-grade neoplasms with areas of increased proliferation. (d) Disseminated low-grade appendiceal mucinous neoplasms (AJCC grade G1) frequently involved other abdominal organs, including dissection into the muscularis propria of the gastrointestinal tract $(\times 20)$. However, these tumors maintained low cytologic grade (inset $\times 400$ ) and lacked any adverse histologic features. (e) A subset of cases demonstrated focal areas concerning for invasion accounting for at most a microscopic focus and were labeled as predominantly low-grade neoplasms with areas of increased proliferation. These areas concerning for invasion were frequently found with tumor involving the wall of other gastrointestinal tract organs $(\times 20)$. (f) The areas concerning for invasion were characterized by focal small mucin pools containing floating clusters of neoplastic mucinous epithelium and/or tubules within the muscularis propria of involved organs $(\times 200)$. Importantly, these areas demonstrated low cytologic grade and no other adverse histologic features were identified elsewhere in the neoplasm. 


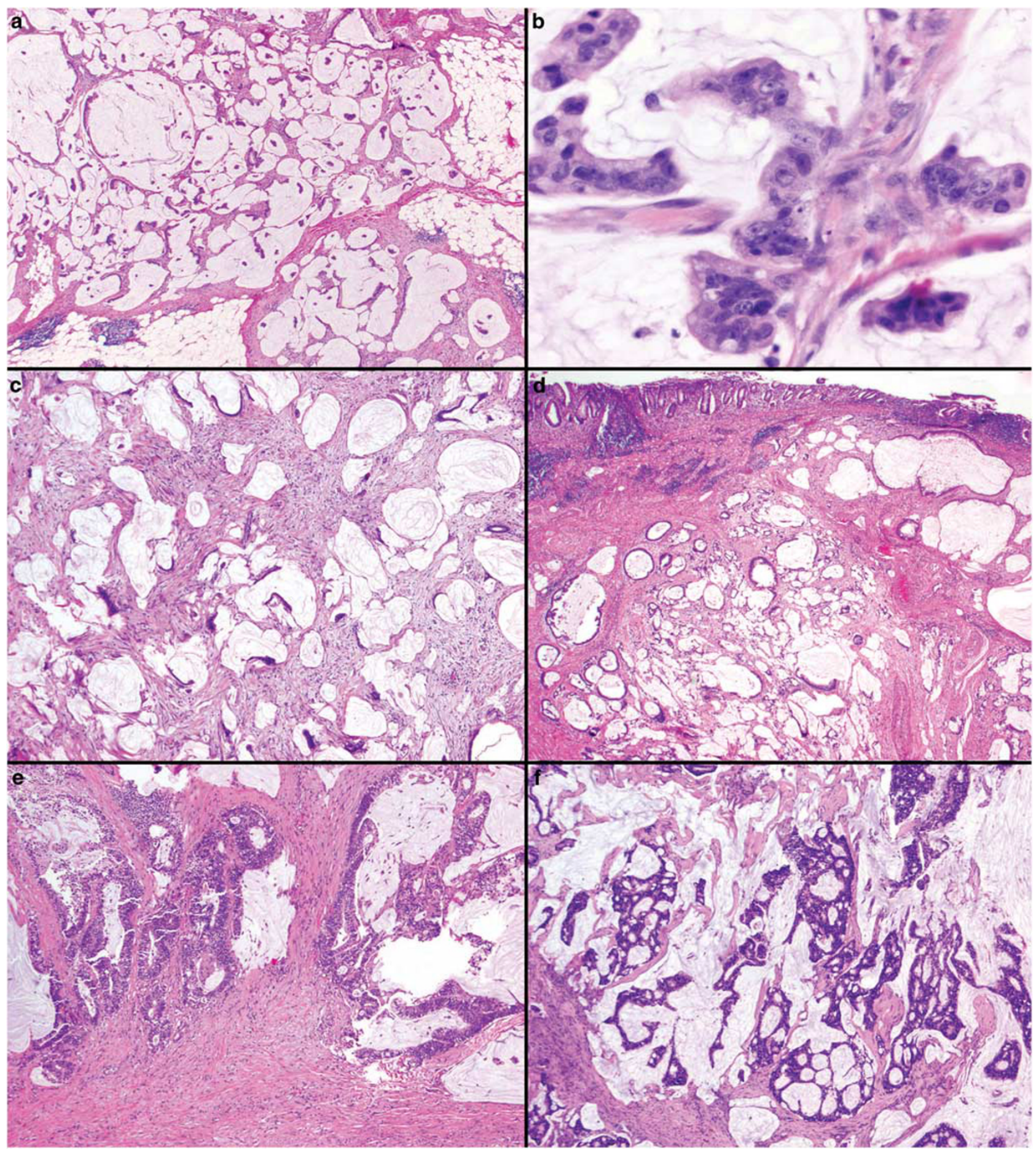

Figure 2 Patterns of invasion in high-grade mucinous adenocarcinomas (AJCC grade G2). (a) This high-grade mucinous adenocarcinoma (AJCC grade G2) involved the omentum and demonstrated destructive invasion with a predominant small mucin pool pattern $(\times 20)$. (b) The neoplastic mucinous epithelium within the small mucin pools demonstrated high cytologic grade $(\times 400)$. (c) This high-grade mucinous adenocarcinoma (AJCC grade G2) extensively involved the peritoneal surfaces and demonstrated a predominant small mucin pool pattern associated with a desmoplastic stromal response $(\times 40)$. (d) This high-grade mucinous adenocarcinoma (AJCC grade G2) extensively infiltrated the wall of the sigmoid colon with both a small mucin pool pattern and infiltrating gland pattern $(\times 20)$. (e) Infiltrating glands associated with a desmoplastic stromal reaction was a less frequently observed predominant pattern of destructive invasion in AJCC grade G2 mucinous adenocarcinoma $(\times 20)$. (f) A complex cribriform architectural pattern was also less frequently observed predominant pattern in AJCC grade G2 mucinous adenocarcinoma $(\times 100)$.

demonstrating high cytologic grade (Figure 3). Angiolymphatic invasion (15\%) and perineural invasion $(5 \%)$ were relatively infrequent and were always accompanied by one or more of the other more commonly observed adverse histologic features. Lymph node involvement was seen in $9 / 54$ 


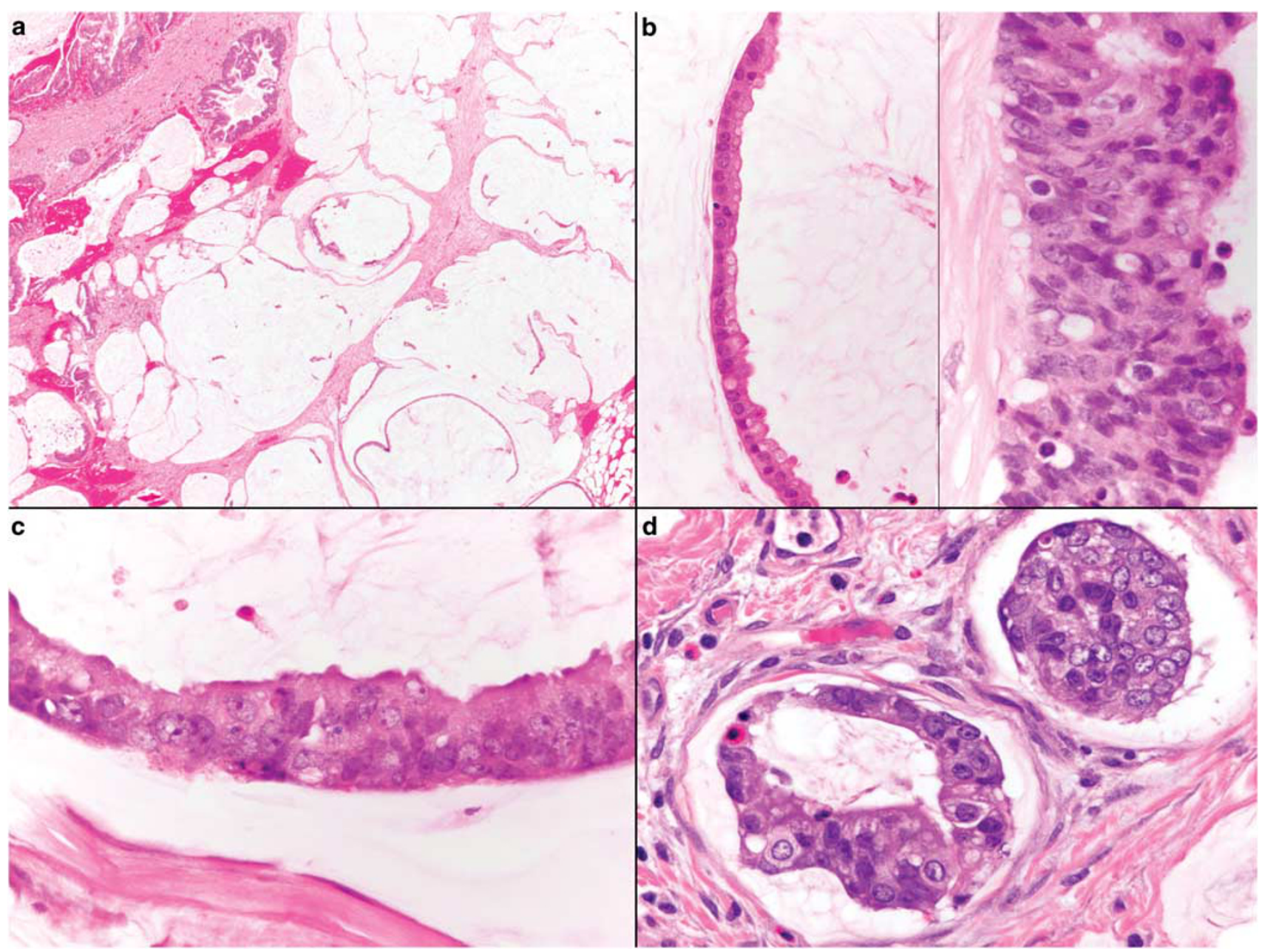

Figure 3 Cytologic grade assessment in AJCC grade G2 mucinous adenocarcinomas. (a) AJCC grade 2 mucinous adenocarcinomas often demonstrated heterogeneity with some areas resembling low-grade mucinous neoplasm (AJCC grade G1) (right half of image) with other areas demonstrating complex architecture (upper left half of image) $(\times 20)$. (b) The adenocarcinoma depicted in part A demonstrated neoplastic epithelium with low cytologic grade (left panel, $\times 400$ ) admixed with neoplastic mucinous epithelium with unequivocal high cytologic grade (right panel, $\times 400$ ). (c) This AJCC grade G2 mucinous adenocarcinoma contained strips of neoplastic epithelium with significant nuclear enlargement, nuclear stratification, prominent nucleoli, and vesicular chromatin sufficient for high cytologic grade ( $\times 400$ ). (d) This AJCC grade G2 mucinous adenocarcinoma had a small mucin pool pattern of invasion with small glands and nests of tumor with nuclear crowding, nuclear enlargement, prominent nucleoli, and vesicular chromatin diffusely present throughout the tumor $(\times 400)$. The degree of cytologic changes is sufficient for a designation of high cytologic grade.

$(17 \%)$ of high-grade mucinous adenocarcinomas, more frequently than AJCC grade 1 tumors $(P=0.005$, Table 2$)$. There were four high-grade mucinous adenocarcinomas with only one adverse histologic feature, three displayed only destructive invasion and one tumor demonstrated only high cytologic grade. There were 12 tumors demonstrating two adverse histologic features, all had both destructive invasion and high cytologic grade.

The small mucin pool pattern was the predominant pattern of invasion in 33/55 (60\%) of high-grade mucinous adenocarcinomas and was characterized by small nests, glands, or single neoplastic cells floating within small pools of mucin with or without a desmoplastic stromal reaction (Figure 2). Infiltrating tubules or single cells with a desmoplastic stromal reaction was the predominant pattern of invasion in 15/55 (27\%) of cases, whereas a complex cribriform growth pattern was observed as the predominant pattern of invasion in 6/55 (11\%) of cases (Figure 2). In addition to the predominant pattern of invasion, 28 cases (51\%) demonstrated secondary pattern(s) of invasion accounting for a minor component of the invasive tumor, most frequently complex cribriform architecture (13 cases) or infiltrating tubules or single cells within a desmoplastic stroma (12 cases).

The primary appendix was available for review in 18 of 55 patients (33\%). Fourteen of 18 primary appendiceal neoplasms demonstrated destructive invasion, although the four primary tumors lacking invasion were not entirely submitted for histologic evaluation. The most common pattern of invasion was the small mucin pool pattern seen in 6/14 (43\%) 
cases while infiltrating tubules with a desmoplastic reaction was the predominant pattern in 5/14 (36\%) and complex cribriform growth was the predominant pattern in $3 / 14(21 \%)$ cases. The same cytologic grade was observed in the primary appendiceal neoplasm and the peritoneal tumor deposits in 16/ $18(89 \%)$ cases. In two cases, the primary neoplasm demonstrated low cytologic grade (Figure 4), whereas the disseminated peritoneal neoplasm was high grade, although the entire appendix was not submitted for histologic review in either case. Angiolymphatic invasion $(3 / 18,17 \%)$ and perineural invasion $(2 / 18,11 \%)$ were infrequently identified in the primary neoplasm.

Pathologic findings in disseminated high-grade mucinous adenocarcinoma with signet ring cells (AJCC Grade G3). A total of 27 patients had a signet ring cell component in their resected tumor (Table 2). The majority $(17 / 27,63 \%)$ of cases were pure signet ring cell carcinoma, composed of $>95 \%$ signet ring cells (Figure 5). The remainder (10/27, $37 \%$ ) contained both mucinous and signet ring cell components: eight cases with between 50 and $95 \%$ signet ring cells and two cases with between 10 and 50\% signet ring cells (Figure 5). All AJCC grade G3 adenocarcinomas demonstrated destructive invasion usually characterized by an infiltrating single cell pattern $(20 / 27,74 \%)$. Compared with AJCC grade G2 adenocarcinomas, grade G3 adenocarcinomas more frequently had lymph node involvement (72 vs $17 \%, P<0.001$ ), angiolymphatic invasion (70 vs $15 \%, P<0.001)$, and perineural invasion (70 vs $5 \%, P<0.001)$.

The primary appendix was available for review in 12/27 cases (44\%). All 12 cases demonstrated infiltrating signet ring cells within the primary appendi- ceal neoplasm. In 11/12 cases, the appendix was almost entirely obliterated with minimal to no residual mucosa present for evaluation of a precursor mucinous lesion; one case had neoplastic mucinous lesion present within the appendiceal mucosa. In 2/12 cases, areas histologically diagnostic of goblet cell carcinoid and confirmed by synaptophysin immunohistochemistry were present associated with the infiltrating signet ring cell adenocarcinoma. An additional four cases without diagnostic histologic features of goblet cell carcinoid had synaptophysin immunohistochemistry performed with two cases demonstrating weak, focal staining within the infiltrating signet ring cells and two cases lacking synaptophysin staining. Angiolymphatic invasion (9/12 cases, $75 \%$ ) and perineural invasion 10/12 (83\%) were quite common in the primary appendiceal tumors associated with AJCC grade G3 metastatic disease.

\section{Comparison of Three-Tier Grading with Original Diagnostic Terminology: Toward Simplified Communication of Prognostic Information}

One of the primary motivations for this study was to simplify the diagnostic terminology so that the pathologic diagnosis would convey all relevant prognostic information to the treating physician. To illustrate how a three-tier grading system based on the presence of histologic risk factors can reduce the complexity of diagnostic terminology, we compared the original diagnostic terminology in use in our own institution over an 8-year period with the final grade from this study (Table 3). A total of 95 specimens from 69 patients were classified as lowgrade mucinous neoplasm (AJCC grade G1) in the current study. The original pathology reports used a

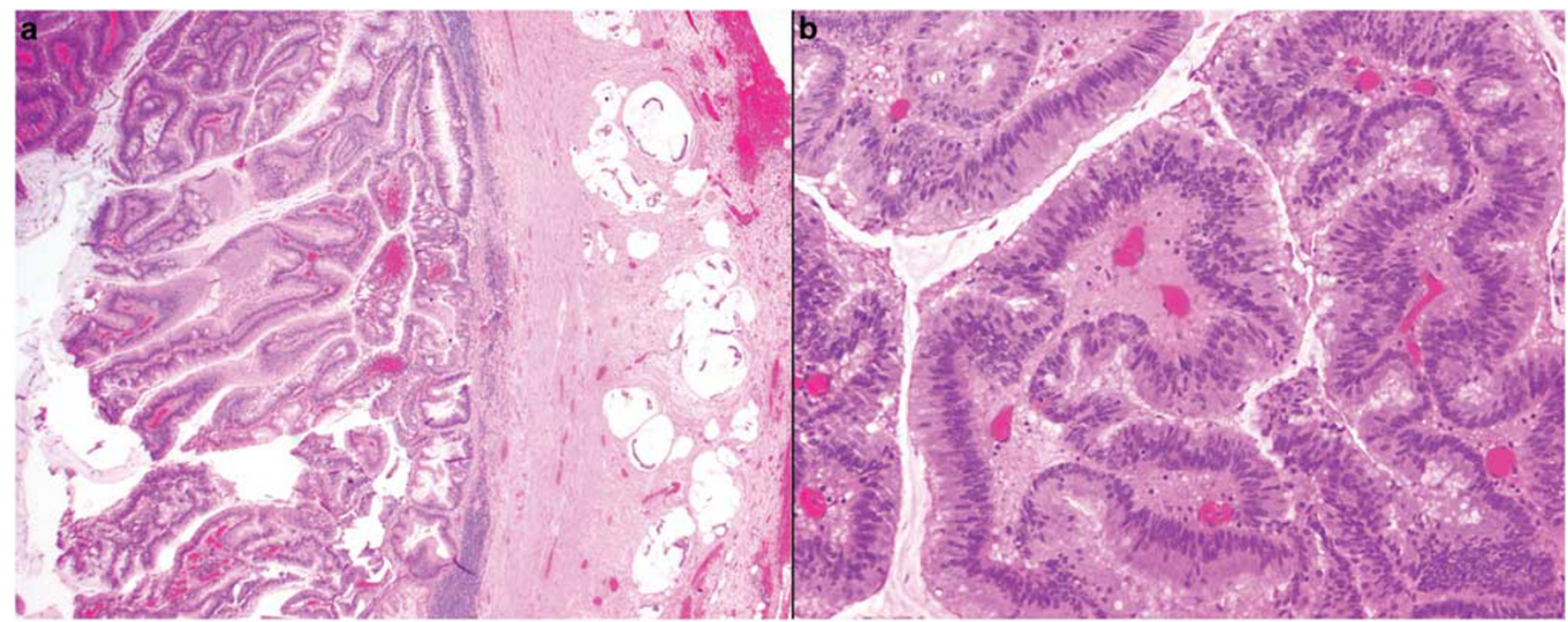

Figure 4 (a) Appendix demonstrating a primary appendiceal mucinous neoplasm with intraluminal villous growth associated with a high-grade (AJCC grade G2) mucinous adenocarcinoma with a small mucin pool pattern of invasion into the appendiceal muscularis propria $(\times 20)$. (b) The appendiceal mucosal precursor lesion demonstrated a villous growth pattern but was characterized by low cytologic grade $(\times 200)$. 


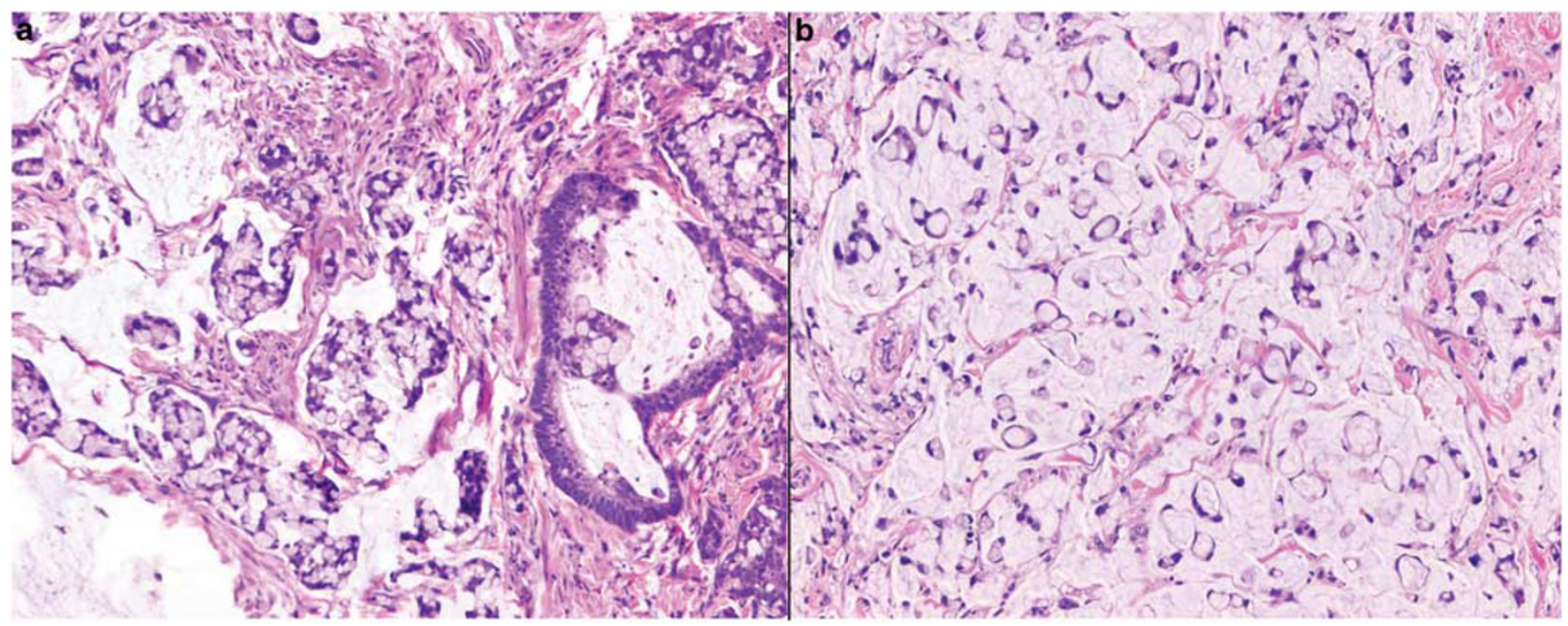

Figure 5 (a) High-grade mucinous adenocarcinoma with signet ring cells (AJCC grade G3) composed of mixed mucinous (right half of image) and signet ring cell components. (b) High-grade mucinous adenocarcinoma with signet ring cells (AJCC grade G3) composed predominantly $(>95 \%)$ of neoplastic signet ring cells associated with copious extracellular mucin $(\times 200)$.

Table 3 Comparison of tumor classification in current study to original diagnostic terminology in the pathology report

Tumor grade classification in current study

\begin{tabular}{|c|c|c|c|}
\hline Diagnostic terminology used in original pathology report & $\begin{array}{l}\text { Grade G1) } \\
\text { N (\%) }\end{array}$ & $\begin{array}{c}\text { (AJCC Grade G2) } \\
\mathrm{N}(\%)\end{array}$ & $\begin{array}{c}\text { (AJCC Grade G3) } \\
\mathrm{N}(\%)\end{array}$ \\
\hline Low-grade mucinous neoplasm or DPAM & $59(62)$ & $3(3)$ & $0(0)$ \\
\hline Well-differentiated/low-grade mucinous adenocarcinoma & $29(31)$ & $33(36)$ & $0(0)$ \\
\hline Moderately-differentiated/intermediate-grade mucinous adenocarcinoma & $3(3)$ & $13(14)$ & $0(0)$ \\
\hline High-grade mucinous adenocarcinoma & $4(4)$ & $24(26)$ & $1(3)$ \\
\hline Mucinous adenocarcinoma, grade not specified & $0(0)$ & $19(21)$ & $0(0)$ \\
\hline High grade/poorly differentiated adenocarcinoma with signet ring cells & $0(0)$ & $0(0)$ & $31(97)$ \\
\hline Total number of individual resection specimens & $95(100)$ & $92(100)$ & $32(100)$ \\
\hline
\end{tabular}

Abbreviations: AJCC, American Joint Committee on Cancer; DPAM, disseminated peritoneal adenomucinosis.

wide variety of diagnostic terminology to classify the initial tumor resection and follow-up surgical resections. Most cases (62\%) we classified as low grade were diagnosed as either low-grade mucinous neoplasms or disseminated peritoneal adenomucinosis. However, $31 \%$ of cases were diagnosed as well-differentiated/low-grade mucinous adenocarcinoma without a clear indication of how this diagnosis differed from a low-grade mucinous neoplasm/disseminated peritoneal adenomucinosis in the diagnostic pathology report. A small subset was diagnosed as moderately differentiated/intermediate-grade adenocarcinoma $(3 \%)$ or high-grade mucinous adenocarcinoma (4\%) based on cytologic grade that was incorrectly classified as high grade.

Including both the initial and follow-up tumor resections, a total of 92 cases were classified as highgrade mucinous adenocarcinoma (AJCC grade G2) in the current study. The most common diagnosis employed in the original pathology report for AJCC grade G2 mucinous adenocarcinomas was welldifferentiated/low-grade mucinous adenocarcinoma (36\%) (Table 3). Twenty-six percent of AJCC grade G2 adenocarcinomas were originally diagnosed as high-grade mucinous adenocarcinomas, whereas $21 \%$ were diagnosed as mucinous adenocarcinoma without any further characterization. Fourteen percent of these cases were classified as moderately differentiated/intermediate-grade mucinous adenocarcinoma. Three tumors $(3 \%)$ were originally diagnosed as low-grade mucinous neoplasms.

Overall, including both the initial and follow-up tumor resections, a total of 32 individual cases were classified as high-grade mucinous adenocarcinoma with signet ring cells (AJCC grade G3) in the current study. In all cases, the original diagnosis was highgrade/poorly differentiated adenocarcinoma with $31 / 32$ cases commenting in the original pathology report on the presence of a signet ring cell component. 


\section{Molecular Analysis of Disseminated Appendiceal Mucinous Neoplasms}

KRAS mutation analysis. KRAS exon 2 mutation analysis was performed in 150 patients (Table 4). KRAS mutation was identified in $61 \%$ of AJCC grade G1 neoplasms and $72 \%$ of AJCC grade G2 adenocarcinomas but in only $19 \%$ of AJCC grade G3 $(P<0.001)$. The KRAS mutations stratified by overall grade were as follows: AJCC grade G1, 28 Gly12Asp, 13 Gly12Val, and 1 Gly13Asp; AJCC grade G2, 23 Gly12Asp, 9 Gly12Val, 2 Gly12Ser, 1 Gly12Cys, and 4 Gly13Asp; and AJCC grade G3: 3 Gly12Asp and 2 Gly12Val. Notably, all AJCC grade G3 adenocarcinomas composed of $>95 \%$ signet ring cells lacked a KRAS mutation.

LOH analysis. The fractional mutation rate is the proportion of chromosomal loci with allelic imbalance (LOH). ${ }^{26}$ Data were available to calculate this metric in 141 patients (Table 4). Most cases (107/141, 76\%) demonstrated a low fractional mutation rate. However, a high fractional mutation rate was more frequently observed in AJCC grade G2 (16/51, 31\%) and grade G3 (9/24, 38\%) adenocarcinomas compared with lowgrade AJCC grade G1 neoplasms $(9 / 66,14 \%)(P=0.02)$.

Two chromosomal loci showed at least a trend toward a higher frequency of LOH in high-grade (AJCC grade G2 or G3) tumors. A significantly higher proportion of AJCC grade G2 (13/51, 25\%) and grade G3 (6/27, 22\%) demonstrated LOH at chromosome $18 \mathrm{q}$ compared with AJCC grade G1 tumors (5/55, $8 \%)(P=0.02)$. At chromosome 17p, 13/51 (25\%) of AJCC grade G2 and 9/33 (22\%) AJCC grade G3 had $\mathrm{LOH}$, compared with $15 \%$ of AJCC grade G1 neoplasms $(P=0.07)$. There was no significant difference in the frequency of $\mathrm{LOH}$ at $1 p, 3 p, 5 q$, $7 q, 9 q, 9 p$, and $10 q$ across tumor grade groups.

\section{Comparison of Treatment Approach and Completeness of Initial Surgery in Patients Stratified by Tumor Grade}

There was no significant difference in the frequency with which patients received hyperthermic intra- peritoneal chemotherapy after surgical debulking when patients were stratified by tumor grade. Surgical debulking with hyperthermic intraperitoneal chemotherapy was performed in 61/69 (88\%) patients with low-grade mucinous neoplasms (AJCC grade G1), in 42/55 (76\%) patients with high-grade (AJCC grade G2) mucinous adenocarcinoma and 21/ $27(77 \%)$ patients with high-grade (AJCC grade G3) mucinous adenocarcinoma with signet ring cells. Surgical debulking without hyperthermic intraperitoneal chemotherapy was performed in 7/69 (10\%) patients with low-grade (AJCC grade G1) disease, 6/ $55(11 \%)$ patients with high-grade (AJCC grade G2) adenocarcinoma, and $3 / 27(11 \%)$ patients with high-grade (AJCC grade G3) adenocarcinoma. There was a slightly higher likelihood of a patient undergoing a limited resection with neither aggressive debulking nor hyperthermic intraperitoneal chemotherapy if they had a high-grade tumor: only $1 /$ 69 (1\%) AJCC grade G1 neoplasm was treated in this way; whereas this treatment was employed for $7 / 55$ $(13 \%)$ AJCC grade G2 adenocarcinomas and 3/27 (11\%) AJCC grade G3 adenocarcinomas $(P=0.04)$.

Data on the macroscopic completeness of the original debulking operation were available in 142/ $151(94 \%)$ of cases (Table 2). All macroscopic disease was resected in the initial operation in approximately half of cases and no significant difference in the frequency of an Ro resection was achieved when comparing patients stratified by tumor grade (Table 2).

\section{Analysis of Overall Survival Differences: Tumor Grade is an Independent Predictor of Overall Survival}

A total of 141 of 151 patients had clinical follow-up data, including 65/69 patients with low-grade (AJCC grade G1) tumors, 50/55 patients with high-grade (AJCC grade G2) mucinous adenocarcinoma, and 26/ 27 patients with high-grade (AJCC grade G3) mucinous adenocarcinoma with signet ring cells. The median follow-up interval was 94 months from the time of initial diagnosis. In the entire cohort,

Table 4 Molecular features of study patients with disseminated appendiceal mucinous neoplasms

\begin{tabular}{|c|c|c|c|c|c|}
\hline Molecular feature & $\begin{array}{c}\text { Low-grade } \\
(\text { AJCC Grade G1) N (\%) }\end{array}$ & $\begin{array}{c}\text { High-grade } \\
\text { (AJCC Grade G2) N (\%) }\end{array}$ & $\begin{array}{c}\text { High-grade } \\
\text { (AJCC Grade G3) N (\%) }\end{array}$ & $\mathrm{P}$-value ${ }^{a}$ & $\mathrm{P}$-value ${ }^{b}$ \\
\hline \multicolumn{6}{|l|}{ KRAS mutation } \\
\hline Not performed & 0 & 1 & 0 & $<0.001$ & $<0.001$ \\
\hline Absent & 27 (39) & $15(28)$ & $22(81)$ & & \\
\hline Present & $42(61)$ & $39(72)$ & 5 (19) & & \\
\hline \multicolumn{6}{|l|}{$\mathrm{LOH}$} \\
\hline Not performed & 3 & 4 & 3 & 0.020 & 0.610 \\
\hline FMR 0-24\% & $57(86)$ & $35(69)$ & $15(63)$ & & \\
\hline $\mathrm{FMR} \geq 25 \%$ & $9(14)$ & $16(31)$ & $9(38)$ & & \\
\hline
\end{tabular}

Abbreviations: AJCC, American Joint Committee on Cancer; FMR, fractional mutation rate; LOH, loss of heterozygosity.

${ }^{\mathrm{a}}$ For the comparison across all three grades (G1, G2, and G3).

$\mathrm{b}_{\mathrm{F}}$ or the comparison of grades $\mathrm{G} 2$ and G3 only. 


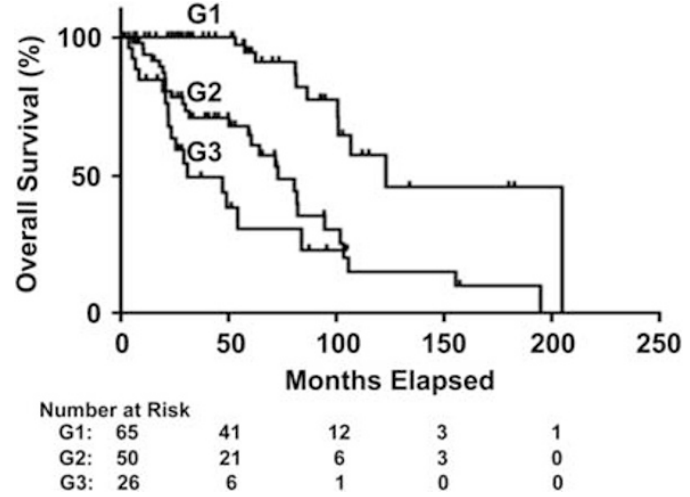

Figure 6 Kaplan-Meier survival curve comparing the overall survival of patients with disseminated low-grade mucinous neoplasm (AJCC grade G1), high-grade mucinous adenocarcinoma (AJCC grade G2), and high-grade mucinous adenocarcinoma with signet ring cells (AJCC grade G3). Patients with AJCC grade G2 and grade G3 mucinous adenocarcinomas had a statistically worse overall survival compared with AJCC grade G1 neoplasms $(P<0.0001$ for each). A trend to reduced survival for patients with AJCC grade G3 mucinous adenocarcinoma compared with patients with AJCC grade G2 mucinous adenocarcinoma was identified $(P=0.07)$.

there were 55 deaths occurring between 3 months and 204 months from the time of initial diagnosis.

Using Kaplan-Meier survival functions to compare patients based on tumor grade, patients with AJCC grade G1 neoplasms had significantly longer overall survival in comparison to patients with AJCC grade G2 mucinous adenocarcinomas $(P<0.0001)$ and AJCC grade G3 mucinous adenocarcinoma with signet ring cells $(P<0.0001)$ (Figure 6). The 5-year survival rate for patients with AJCC grade G1 neoplasms was 91\%, compared with $61 \%$ for patients with AJCC grade G2 adenocarcinoma and $23 \%$ for patients with AJCC grade G3 adenocarcinoma with signet ring cells. When comparing high-grade (grades G2 and G3) tumors only, we saw a statistical trend toward worse survival outcomes for patients with AJCC grade G3 mucinous adenocarcinoma with signet ring cells compared with AJCC grade G2 mucinous adenocarcinomas $(P=0.07)$.

Patients with AJCC grade G2 mucinous adenocarcinoma and AJCC grade G3 mucinous adenocarcinoma with signet ring cells had a significantly shorter time to progression compared with AJCC grade G1 neoplasms $(P<0.001$ for each). No significant difference in time to progression was identified between patients with AJCC grade G2 and AJCC grade G3 adenocarcinomas $(P=0.20$, log rank test).

Using Cox proportional hazards modeling, we evaluated the association of pathologic, molecular, and clinical factors with survival after initial therapy (Table 5). High tumor grade was associated with a 4.6-fold (AJCC grade G2) or 8.2-fold (AJCC grade G3) increased risk of death relative AJCC grade G1. Lymph node involvement was also a poor pro- gnostic factor in the univariate analysis $(P<0.001)$. At the molecular level, a high fractional mutation rate was a significant adverse risk factor for overall survival $(P=0.001)$, but the presence of KRAS mutation was not. Of the clinical variables we evaluated, use of hyperthermic intraperitoneal chemotherapy therapy was associated with a $70 \%$ reduction in the risk of death $(P<0.001)$ in the univariate analysis. The presence of any macroscopic residual disease did not influence survival. Increasing age and male sex were borderline risk factors (Table 5).

In the multivariable analysis that included all variables with at least borderline association with survival $(P<0.1)$ from the univariate analysis, only AJCC grade was an independent predictor of overall survival (Table 5). A diagnosis of AJCC grade G2 was associated with a 2.7-fold increased risk of death and a diagnosis of AJCC grade G3 was associated with a 5.1-fold increased risk of death when controlling for patient age, sex, lymph node involvement, high fractional mutation rate, and use of hyperthermic intraperitoneal chemotherapy at initial treatment (Table 5).

\section{Fractional Mutation Rate May Identify Differences in Outcome in High-Grade (Grades G2 and G3) Mucinous Adenocarcinomas}

Tumors with a high frequency of chromosomal LOH (fractional mutation rate $\geq 25 \%$ ) had a 2.8 -fold increased risk of death in comparison to those with a low fractional mutation rate in univariate analysis $(P=0.001)$, but the multivariable analysis showed that its influence on survival is not independent of other risk factors (Table 5). We therefore considered whether this molecular characteristic could be combined with AJCC grade to predict survival in patients with high-grade tumors (AJCC grades G2 or G3) due to their borderline difference in survival. We found that among high-grade tumors, a high fractional mutation rate was associated with poor prognosis (HR, 2.0; 95\% confidence interval (CI), 1.04-3.85, $P=0.039$ ). Hyperthermic intraperitoneal chemotherapy was also associated with better prognosis in the high-grade subgroup (HR, 0.44; 95\% CI, 0.22-0.88). The limited number of cases precluded a multivariable analysis of prognostic factors in the high-grade subgroup.

\section{Clinical Outcome of Relevant Histopathologic Subgroups}

Disseminated low-grade mucinous neoplasms (AJCC Grade G1) with progression to high-grade mucinous adenocarcinoma (AJCC Grade G2). Seven patients initially presented with AJCC grade G1 neoplasms but subsequently developed progressive disease and had high-grade (AJCC grade G2) mucinous adenocarcinoma diagnosed on subsequent surgical 
Table 5 Univariate and multivariate analysis of overall survival according to histologic, molecular, and clinical features

\begin{tabular}{|c|c|c|c|c|c|}
\hline \multirow[b]{2}{*}{ Histologic or molecular feature } & \multirow[b]{2}{*}{ Patients } & \multicolumn{2}{|c|}{ Univariate analysis } & \multicolumn{2}{|c|}{ Multivariate analysis ${ }^{a}$} \\
\hline & & Hazard ratio (95\% CI) & $\mathrm{P}$-value & Hazard ratio (95\% CI) & $\mathrm{P}$-value \\
\hline \multicolumn{6}{|l|}{ Overall tumor grade } \\
\hline Grade G1 & 65 & Referent & $<0.001$ & Referent & 0.008 \\
\hline Grade G2 & 50 & $4.6(2.2-9.5)$ & & $2.7(1.2-6.2)$ & \\
\hline Grade G3 & 26 & $8.2(3.7-18.4)$ & & $5.1(1.7-14)$ & \\
\hline \multicolumn{6}{|l|}{ Lymph node involvement } \\
\hline Absent & 111 & Referent & $<0.001$ & Referent & 0.126 \\
\hline Present & 27 & $3.3(1.8-6.0)$ & & $1.8(0.8-4.1)$ & \\
\hline \multicolumn{6}{|l|}{ KRAS mutation } \\
\hline Absent & 62 & Referent & 0.692 & _- & - \\
\hline Present & 78 & $0.9(0.5-1.6)$ & & & \\
\hline \multicolumn{6}{|l|}{$\mathrm{LOH}$} \\
\hline FMR 0-24\% & 98 & Referent & 0.001 & Referent & 0.371 \\
\hline $\mathrm{FMR} \geq 25 \%$ & 33 & $2.8(1.5-5.2)$ & & $1.4(0.6-3.0)$ & \\
\hline Patient age, years & 151 & $1.0(0.99-1.05)$ & 0.059 & $1.0(0.97-1.03)$ & 0.941 \\
\hline \multicolumn{6}{|l|}{ Sex } \\
\hline Male & 73 & Referent & 0.085 & Referent & 0.099 \\
\hline Female & 68 & $0.6(0.4-1.1)$ & & $0.6(0.3-1.1)$ & \\
\hline \multicolumn{6}{|l|}{ Macroscopic residual disease } \\
\hline R0 & 71 & Referent & 0.84 & - & - \\
\hline R1 & 64 & $0.94(0.5-1.7)$ & & & \\
\hline \multicolumn{6}{|l|}{ HIPEC therapy } \\
\hline Not given & 20 & Referent & $<0.001$ & Referent & 0.112 \\
\hline Given & 121 & $0.3(0.2-0.6)$ & & $0.5(0.2-1.2)$ & \\
\hline
\end{tabular}

Abbreviations: CI, confidence interval; FMR, fractional mutation rate; HIPEC, hyperthermic intraperitoneal chemotherapy; LOH, loss of heterozygosity.

${ }^{a}$ Variables for which $P<0.1$ were included in the multivariable model.

resections. None of the AJCC grade G1 in our series progressed to AJCC grade G3 adenocarcinomas with a signet ring cell component. Destructive invasion was a histologic finding that warranted a diagnosis of AJCC grade G2 in all seven patients; high cytologic grade contributed to the diagnosis in three patients and high tumor cellularity in four patients. The time to progression to AJCC grade G2 mucinous adenocarcinoma ranged from 9 months to 43 months. Of the seven patients, two died of disease at 101 months and 86 months after presentation with the remaining five patients alive at last followup. However, patients with grade G1 to G2 progression had no significant difference in overall survival compared with patients with stable low-grade disease with recurrence or no recurrence (HR 1.1; 95\% CI 0.5-2.3). Although, by definition all those with progression to AJCC grade G2 mucinous adenocarcinoma had disease progression, the time to progression was accelerated in patients who developed high-grade disease in comparison to patients with low-grade disease who progressed with stable tumor grade (HR 1.9; 95\% CI 1.3-3.0).

Disseminated low-grade mucinous neoplasms (AJCC Grade G1) with increased proliferation. Eight patients had tumors which were predominantly low-grade but exhibited focal areas of increased proliferation. Clinical follow-up was available for seven patients. None of the seven patients progressed to high-grade mucinous adenocarcinoma during the follow-up period or died of disease at last clinical follow-up. However, four of seven patients $(57 \%)$ developed tumor recurrence by 3 years after the initial surgical debulking operation. Patients with AJCC grade G1 neoplasms with increased proliferation had a trend to accelerated time to progression compared with patients with other low-grade neoplasms, although this did not reach statistical significance (HR 2.4; 95\% CI 0.827.2). Given the small number of cases, we are unable to determine whether there are other factors confounding this association (eg presence of residual tumor after initial debulking and hyperthermic intraperitoneal chemotherapy).

Disseminated low-grade mucinous neoplasms (AJCC Grade G1) with acellular mucin only. Of the 65 patients with low-grade mucinous neoplasms (G1), 5 had peritoneal tumor composed entirely of acellular mucin and none of the 5 patients died or 
developed recurrent disease at last follow-up (median follow-up 32 months, range 1-44 months).

High-grade mucinous adenocarcinoma (AJCC Grade G2) and the number of adverse histologic features. When considering all cases (including AJCC grades G1, G2, and G3), the total number of unfavorable or adverse histologic characteristics (high cytologic grade, destructive, invasion, signet ring cells, angiolymphatic invasion, and perineural invasion) was a strong adverse prognostic factor, similar to AJCC grade $(P<0.001$, data not shown). Although there was considerable variation in the number of adverse histologic characteristics among AJCC grade G2 mucinous adenocarcinomas, the number of adverse histologic features did not significantly affect overall survival or time to progression. In other words, patients with AJCC grade 2 mucinous adenocarcinoma with one or two adverse histologic features had a similarly reduced overall survival compared with patients with AJCC grade G2 mucinous adenocarcinomas with $\geq 3$ adverse histologic features.

Mixed high-grade (AJCC Grade G3) versus pure signet ring cell adenocarcinoma (AJCC Grade G3). The high-grade mucinous adenocarcinoma subgroup with signet ring cells (AJCC grade G3) was heterogeneous with respect to the fraction of the tumor exhibiting signet ring cell features. Although AJCC grade G3 carried a poor prognosis relative to AJCC grade G1 (Table 5), there was no significant difference in overall survival or time to progression between patients who had mixed mucinous/signet ring cell adenocarcinoma $(N=11)$ and patients who had pure $(>95 \%)$ signet ring cell adenocarcinoma ( $N=15),(P>0.05$ for both).

\section{Discussion}

The purpose of our study was not to introduce another classification system for appendiceal mucinous neoplasms and add to the existing confusion in the literature. Instead, the intent of our analysis was to evaluate histopathologic criteria for the threetiered AJCC grading system for disseminated mucinous appendiceal neoplasms and validate its prognostic utility. For the most part, we evaluated prognostically unfavorable histologic characteristics that have been described in the literature. We validated the prognostic significance of these individual criteria and then proposed definitions of AJCC grade G1, G2 and G3 for disseminated appendiceal mucinous neoplasms based on these criteria. The results of our analysis demonstrate that evaluation and reporting of these adverse histologic features allows for the separation of disseminated mucinous neoplasms into three morphologically defined prognostic groups as adopted by the AJCC.
The distinction between low-grade (AJCC grade G1) and high-grade (AJCC grades G2 and G3) tumors is of critical importance in guiding patient therapy. At our institution, patients with low-grade (grade G1) mucinous neoplasms are generally treated with a combined modality strategy including surgical cytoreduction and intraperitoneal chemotherapy. In contrast, those patients with highgrade (grades G2 or G3) mucinous adenocarcinomas are often given systemic neoadjuvant chemotherapy in an attempt to reduce tumor volume with the option for cytoreductive surgery and intraperitoneal chemotherapy after assessment of tumor response to systemic chemotherapy. ${ }^{22}$ Our current study highlights that most pathologists continue to have difficulty in subclassifying disseminated appendiceal neoplasms into prognostically relevant categories. The most difficult distinction is between low-grade (grade G1) mucinous neoplasm and high-grade (grade G2) mucinous adenocarcinoma. In our study patients, the original diagnostic pathology reporting demonstrated significant overlap of the reported diagnosis between tumors re-classified as low grade (grade G1) and high grade (grade G2) with no clear description of the histologic features used in establishing the diagnosis in the original pathology reports. The AJCC uses standard colorectal diagnostic terminology (well-, moderately-, and poorly differentiated) to describe the three grades of mucinous appendiceal neoplasms. Standard grading of colorectal and other gastrointestinal malignancies is almost entirely dependent upon tumor architecture. For instance, the presence of solid or single-cell infiltrating growth patterns within $>50 \%$ of the tumor is required to classify an adenocarcinoma as high grade, according to the WHO. Our data suggests that this system is inadequate for appendiceal mucinous neoplasms because important morphologic variables are not considered. On the basis of the clinical outcome data of the patients in our study, the evaluation for and reporting of a limited number of histologic features including destructive invasion, cytologic grade, tumor cellularity, angiolymphatic and perineural invasion, and signet ring cells allows for the separation of disseminated appendiceal mucinous into the three prognostically relevant groups as adopted by the AJCC. Importantly, we demonstrate that separation of disseminated appendiceal mucinous neoplasms into the three AJCC grades is highly reproducible as these adverse histologic features often co-vary. That is, neoplasms with high cytologic grade will often demonstrate other adverse histologic features such as invasion and high tumor cellularity. Our results indicate that the convincing presence of at least one adverse histologic feature is sufficient to label the neoplasm as high-grade mucinous adenocarcinoma and that this designation conveys a significant risk of aggressive clinical behavior and worse overall survival. 


\section{Low-Grade Mucinous Neoplasm/Adenocarcinoma (AJCC Grade G1)}

Low-grade mucinous neoplasm/adenocarcinoma, AJCC grade G1, would encompass those cases previously classified as disseminated peritoneal adenomucinosis by Ronnett et $a 1^{10,11}$ and those cases classified as low-grade mucinous carcinoma peritonei by Bradley et al. ${ }^{7}$ Low-grade mucinous neoplasm and low-grade mucinous adenocarcinoma are equally acceptable and interchangeable diagnostic terms. In our practice, we have elected to employ low-grade mucinous neoplasm as the preferred diagnostic terminology to avoid confusion with gland-forming high-grade adenocarcinomas. Regardless of the terminology employed, the important information to be conveyed is the absence of any adverse histologic features in lowgrade (AJCC grade G1) tumors. The overall 5-year survival for patients with low-grade tumors in our series of $91 \%$ is slightly higher than the $63-86 \%$ range reported in the literature,,$^{6,7,9-11}$ although direct comparisons are difficult given the heterogeneous therapeutic protocols employed and the inclusion of non-appendiceal primary neoplasms in some studies. ${ }^{10,11}$ The 10 -year survival in our series of $46 \%$ is similar to the $45-$ $68 \%$ rate reported in the literature. ${ }^{9,10,15}$

When evaluating for adverse histologic features, the most difficult histologic assessments are for (1) destructive invasion within neoplasms that have already widely disseminated within the peritoneal cavity and (2) cytologic grade. Low-grade tumors frequently dissect into the wall of intestinal organs and involve the spleen, pancreas, ovaries, omentum, and liver parenchyma. However, the presence of neoplastic mucinous epithelium and mucin within these organs is not sufficient for a diagnosis of destructive invasion as these tumors typically display a 'pushing' border without unequivocal, haphazard infiltration. Nuclear grade can also be difficult to assess in disseminated mucinous neoplasms for a variety of reasons including (1) associated inflammatory reaction raising the possibility of superimposed reactive epithelial changes versus high cytologic grade, (2) heterogeneity of nuclear features within a given tumor, and (3) nuclear features which span the spectrum of lowand high-grade making definitive assessment of grade difficult. In our series, $12 \%$ of low-grade tumors had either a questionable microscopic area of destructive invasion or nuclear atypia beyond that typical for low nuclear grade but insufficient for an unequivocal label of high-nuclear grade accounting for $<10 \%$ of the tumor. This subgroup of patients likely corresponds to the peritoneal mucinous carcinomatosis-intermediate category described by Bradley et al. ${ }^{7}$ Bradley et al identified 20 patients with peritoneal mucinous carcinomatosis-intermediate category and found no significant difference in overall survival between patients with peritoneal mucinous carcinomatosis-intermediate category and patients with disseminated peritoneal adenomucinosis. ${ }^{7}$ Similarly, in our series, there was no significant difference in overall survival for this group of patients compared with other patients with low-grade mucinous neoplasms. Ronnett et al $1^{10,11}$ also identified a subgroup of patients with peritoneal mucinous carcinomatosis-intermediate category; however, their results are difficult to compare with our series as non-appendiceal mucinous neoplasms were also included in their analysis and patients with discrepant histology were combined with patients with peritoneal mucinous carcinomatosis-intermediate category in their survival analysis. In our practice, we have provisionally labeled tumors with questionable microscopic areas of invasion and/or focal nuclear atypia beyond that typical for low cytologic grade as predominantly low grade (AJCC grade G1) with focal areas of increased proliferation and provide a descriptive comment on the histologic features warranting this diagnosis. These patients may benefit from close clinical surveillance as our data suggest that they may recur more rapidly.

In our series, $7 \%$ of patients with low-grade mucinous neoplasms had disseminated appendiceal mucinous neoplasms composed entirely of acellular mucinous deposits within the peritoneal cavity despite extensive histologic sampling and evaluation for neoplastic mucinous epithelium. None of these patients developed recurrent disease during clinical follow-up suggesting that these neoplasms have a relatively indolent clinical course. In their analysis, Young et $a l^{5}$ identified five patients with acellular peritoneal disease; four of the patients were without evidence of disease recurrence at last follow-up (range 1-8 years). One patient in their series developed disease recurrence 18 years after initial presentation. Along with our data, the limited literature data suggest that patients with acellular peritoneal mucin have a much lower risk of disease recurrence and have an improved overall survival compared with those patients with cellular low-grade disease.

\section{High-Grade Mucinous Adenocarcinoma (AJCC Grade G2)}

High-grade mucinous adenocarcinoma demonstrates at least one adverse histologic feature but lacks a signet ring cell component. In our series, patients with high-grade mucinous adenocarcinoma (AJCC grade G2) had a significantly worse overall survival in comparison to patients with low-grade (AJCC grade G1) tumors. The overall 5-year survival in our series of $61 \%$ is slightly higher than the $23-$ $44 \%$ reported in the literature;,6,7-11 however, these prior literature reports combined adenocarcinomas with and without a signet ring cell component into one diagnostic category precluding a direct comparison. Our results demonstrate that the number of 
adverse histologic features identified within a neoplasm does not alter prognosis as no difference in overall survival was identified between patients with adenocarcinomas demonstrating one or two adverse histologic features and those with adenocarcinomas demonstrating $\geq 3$ adverse histologic features.

The most frequent adverse histologic features identified were destructive invasion (98\%), high cytologic grade (87\%), and high tumor cellularity $(75 \%)$. Histologic assessment for destructive invasion within disseminated neoplasms can be difficult. A unique pattern of invasion in appendiceal mucinous adenocarcinoma that is often overlooked is the presence of small epithelial clusters with or without tubule formation floating in small pools of mucin invading through the subjacent normal tissues but not necessarily eliciting stromal desmoplasia. In our series, this pattern of invasion was identified in the majority $(60 \%)$ of high-grade mucinous adenocarcinomas. Cytologic grade within a disseminated appendiceal mucinous neoplasms can be heterogeneous with areas of low cytologic grade admixed with areas of unequivocal high cytologic grade, and this heterogeneity highlights the need for generous sampling of peritoneal tumor deposits for histologic evaluation. Finally, we assessed for tumor cellularity using a cut point of $\geq 20 \%$ to label tumors as highly cellular. With the exception of Misdraji and colleagues who qualitatively described disseminated tumors as scant to moderately cellular versus highly cellular, assessment of tumor cellularity has not been included in most previous clinicopathologic studies of disseminated appendiceal mucinous neoplasia. .,9,11 $^{2}$ Assessment of overall cellularity is best performed with review of the entire case and is best determined at low-power $(\times 20)$ magnification. In our series, the distinction between low- and high-cellularity neoplasms was not usually difficult. Neoplasms with high cellularity demonstrated numerous epithelial cell clusters within mucin pools easily visible at low-power magnification, as depicted in Figure 2a. The low-power assessment of cellularity is often the first histologic clue to the diagnosis of a high-grade (grade G2) mucinous adenocarcinoma. Importantly, in our series, all high-grade (AJCC grade G2) mucinous adenocarcinoma demonstrating high cellularity also demonstrated destructive invasion and/or high cytologic grade.

\section{High-Grade Mucinous Adenocarcinoma with Signet Ring Cells (AJCC Grade G3)}

This group represents a heterogeneous group of appendiceal adenocarcinomas that have in common the presence of a signet ring cell component. Compared with patients with high-grade mucinous adenocarcinoma without a signet ring cell component (AJCC grade G2), patients with high-grade mucinous adenocarcinoma with signet ring cells had a trend toward worse overall survival. Most tumors $(63 \%)$ that we included in the AJCC grade G3 category were composed of $>95 \%$ signet ring cells. A minority of cases (37\%) displayed a mixed mucinous and signet ring cell morphology with $>10 \%$ signet ring cell component with infiltration into subjacent normal tissues. Although interobserver agreement on overall grade was very high, the presence of focal areas ( $<10 \%$ of the tumor area) with signet ring cell-like morphology often floating in mucin pools was the most frequent reason for a discordant grade assessment between AJCC grade G2 and grade G3. Because these were small foci that could represent degenerating neoplastic mucinous epithelial cells rather than a true signet ring cell component, we elected to assign an overall AJCC grade G2 to such neoplasms. Supporting the inclusion of these cases in the AJCC grade G2 category is the frequent presence of a KRAS mutation in these tumors $(3 / 3,100 \%)$ which was often seen in AJCC grade G2 adenocarcinomas but infrequently identified in AJCC grade G3 adenocarcinomas. Our experience suggests that it may be helpful for the purposes of diagnostic reproducibility to require more than focal (eg $>10 \%$ ) signet ring cells to qualify as AJCC grade G3.

Two patients in our series had metastatic tumor deposits composed of pure ( $>95 \%$ ) signet ring cells and also harbored a primary appendiceal adenocarcinoma associated with a goblet cell carcinoid (mixed adenoneuroendocrine carcinoma or adenocarcinoma ex goblet cell carcinoid). ${ }^{24}$ This suggests that goblet cell carcinoids may represent the precursor lesion for a subset of these adenocarcinomas. Supporting this notion is our finding that relatively few adenocarcinomas with signet ring cells in our study had mutations in KRAS in comparison to AJCC grade G2 adenocarcinomas. Previous studies have shown that goblet cell carcinoid tumors seldom harbor KRAS mutations. $^{27,28}$ Unfortunately, in most cases in our series, the appendix was entirely obliterated by infiltrating adenocarcinoma precluding assessment for a precursor lesion.

\section{Molecular Comparison of Disseminated Appendiceal Mucinous Neoplasms by AJCC Grade}

Our analysis demonstrates that KRAS mutations are frequently identified in $61 \%$ of disseminated lowgrade (grade G1) mucinous neoplasms and $72 \%$ of high-grade mucinous adenocarcinoma (grade G2), most frequently in codon 12. Most previous studies have identified KRAS mutation in a high proportion of disseminated low-grade mucinous neoplasms., ${ }^{4,29}$ The frequent presence of activating KRAS mutations in disseminated mucinous neoplasms arising from the appendix is not surprising given that KRAS mutations have been identified in between 41 and 
$100 \%$ appendiceal mucinous adenomas ${ }^{4,29,30}$ which are the presumed precursor lesion to disseminated neoplasms. Notably, none of the high-grade mucinous adenocarcinomas with signet ring cells (grade G3) composed of a pure (>95\%) signet ring cell component harbored a KRAS mutation suggesting that the underlying genetic events leading to carcinogenesis are different for grade G3 adenocarcinomas.

We have routinely performed $\mathrm{LOH}$ analysis on disseminated appendiceal mucinous neoplasms at our institution, and our treating clinicians have used the fractional mutation rate, along with pathologic classification, to assess prognosis based on the results of a previous retrospective study. ${ }^{26}$ Our current, prospectively collected molecular data confirm the results from the previous study: highgrade mucinous adenocarcinomas (grades G2 and G3) more frequently accumulate allelic loss and mutational damage compared with low-grade (Grade G1) tumors. We found that the presence of high frequency $\mathrm{LOH}$ (fractional mutation rate $\geq 25 \%$ ) is also associated with poor survival. Although this difference in survival was not independent of overall AJCC tumor grade, our data suggest that quantification of genomic alterations (aneuploidy) may help identify differences in prognosis among high-grade tumors.

In summary, evaluation for and reporting of a limited number of adverse histologic features allows for the separation of disseminated mucinous neoplasms into the three-tiered classification adopted by the AJCC. Regardless of the eventual diagnostic terminology employed, this limited set of histologic features is important information that should be conveyed to treating clinicians in diagnostic pathology reports. Our results demonstrate that the overall AJCC grade (G1, G2, and G3) that is based on assessment for adverse histologic features is the single most important prognostic factor for patients. At our institution, we have elected to include a description of these histologic features in all of our diagnostic reporting of disseminated appendiceal mucinous neoplasms in an effort to standardize our diagnostic reporting and to minimize confusion among pathologists and treating clinicians.

\section{Disclosure/conflict of interest}

The authors declare no conflict of interest.

\section{References}

1 Chuaqui RF, Zhuang Z, Emmert-Buck MR, et al. Genetic analysis of synchronous mucinous tumors of the ovary and appendix. Hum Pathol 1996;27:165-171.

2 Ronnett BM, Shmookler BM, Diener-West M, et al. Immunohistochemical evidence supporting the appendiceal origin of pseudomyxoma peritonei in women. Int J Gynecol Pathol 1997;16:1-9.
3 Teixeira MR, Qvist H, Giercksky KE, et al. Cytogenetic analysis of several pseudomyxoma peritonei lesions originating from a mucinous cystadenoma of the appendix. Cancer Genet Cytogenet 1997;93: 157-159.

4 Szych C, Staebler A, Connolly DC, et al. Molecular genetic evidence supporting the clonality and appendiceal origin of Pseudomyxoma peritonei in women. Am J Pathol 1999;154:1849-1855.

5 Young RH, Gilks CB, Scully RE. Mucinous tumors of the appendix associated with mucinous tumors of the ovary and pseudomyxoma peritonei. A clinicopathological analysis of 22 cases supporting an origin in the appendix. Am J Surg Pathol 1991;15:415-429.

6 Carr NJ, Finch J, Ilesley IC, et al. Pathology and prognosis in pseudomyxoma peritonei: a review of 274 cases. J Clin Pathol 2012;65:919-923.

7 Bradley RF, JHt Stewart, Russell GB, et al. Pseudomyxoma peritonei of appendiceal origin: a clinicopathologic analysis of 101 patients uniformly treated at a single institution, with literature review. Am J Surg Pathol 2006;30:551-559.

8 Pai RK, Beck AH, Norton JA, et al. Appendiceal mucinous neoplasms: clinicopathologic study of 116 cases with analysis of factors predicting recurrence. Am J Surg Pathol 2009;33:1425-1439.

9 Misdraji J, Yantiss RK, Graeme-Cook FM, et al. Appendiceal mucinous neoplasms: a clinicopathologic analysis of 107 cases. Am J Surg Pathol 2003;27: 1089-1103.

10 Ronnett BM, Yan H, Kurman RJ, et al. Patients with pseudomyxoma peritonei associated with disseminated peritoneal adenomucinosis have a significantly more favorable prognosis than patients with peritoneal mucinous carcinomatosis. Cancer 2001;92:85-91.

11 Ronnett BM, Zahn CM, Kurman RJ, et al. Disseminated peritoneal adenomucinosis and peritoneal mucinous carcinomatosis. A clinicopathologic analysis of 109 cases with emphasis on distinguishing pathologic features, site of origin, prognosis, and relationship to "pseudomyxoma peritonei". Am J Surg Pathol 1995; 19:1390-1408.

12 Baratti D, Kusamura S, Nonaka D, et al. Pseudomyxoma peritonei: clinical pathological and biological prognostic factors in patients treated with cytoreductive surgery and hyperthermic intraperitoneal chemotherapy (HIPEC). Ann Surg Oncol 2008;15: 526-534.

13 Carr NJ, McCarthy WF, Sobin LH. Epithelial noncarcinoid tumors and tumor-like lesions of the appendix. A clinicopathologic study of 184 patients with a multivariate analysis of prognostic factors. Cancer 1995;75:757-768.

14 Pai RK, Longacre TA. Appendiceal mucinous tumors and pseudomyxoma peritonei: histologic features, diagnostic problems, and proposed classification. Adv Anat Pathol 2005;12:291-311.

15 Chua TC, Moran BJ, Sugarbaker PH, et al. Early- and long-term outcome data of patients with pseudomyxoma peritonei from appendiceal origin treated by a strategy of cytoreductive surgery and hyperthermic intraperitoneal chemotherapy. J Clin Oncol 2012;30: 2449-2456.

16 Yan TD, Black D, Savady R, et al. A systematic review on the efficacy of cytoreductive surgery and perioperative intraperitoneal chemotherapy for pseudomyxoma peritonei. Ann Surg Oncol 2007;14:484-492. 
17 Bryant J, Clegg AJ, Sidhu MK, et al. Systematic review of the Sugarbaker procedure for pseudomyxoma peritonei. Br J Surg 2005;92:153-158.

18 McBride K, McFadden D, Osler T. Improved survival of patients with pseudomyxoma peritonei receiving intraperitoneal chemotherapy with cytoreductive surgery: a systematic review and meta-analysis. J Surg Res 2013;183:246-252.

19 Cole KL, Choudry HA, Jones HL, et al. Critical role of hyperthermic intraperitoneal chemoperfusion in the treatment of a patient with Pseudomyxoma peritonei. J Surg Oncol 2012;106:513-516.

20 Elias D, Gilly F, Quenet F, et al. Pseudomyxoma peritonei: a French multicentric study of 301 patients treated with cytoreductive surgery and intraperitoneal chemotherapy. Eur J Surg Oncol 2010;36:456-462.

21 Shapiro JF, Chase JL, Wolff RA, et al. Modern systemic chemotherapy in surgically unresectable neoplasms of appendiceal origin: a single-institution experience. Cancer 2010;116:316-322.

22 Bijelic L, Kumar AS, Stuart OA, et al. Systemic chemotherapy prior to cytoreductive surgery and HIPEC for carcinomatosis from appendix cancer: impact on perioperative outcomes and short-term survival. Gastroenterol Res Pract 2012;2012:163284.

23 Sugarbaker PH, Bijelic L, Chang D, et al. Neoadjuvant FOLFOX chemotherapy in 34 consecutive patients with mucinous peritoneal carcinomatosis of appendiceal origin. J Surg Oncol 2010;102:576-581.
24 Carr NJ, Sobin LH. Adenocarcinoma of the appendix, In: Bosman FT, Carneiro CF, Hruban RH, Theise ND(eds) WHO Classiciation of Tumours of the Digestive System. IARC Press: Lyon, France; 2010, pp 122-125.

25 Edge SB, Byrd DR, Compton CC, Fritz AG, Greene FL, Trotti A (eds). AJCC Cancer Staging Manual. 7th Ed.. Springer: Chicago, IL; 2010, pp 133-141.

26 Maheshwari V, Tsung A, Lin Y, et al. Analysis of loss of heterozygosity for tumor-suppressor genes can accurately classify and predict the clinical behavior of mucinous tumors arising from the appendix. Ann Surg Oncol 2006;13:1610-1616.

27 Stancu M, Wu TT, Wallace C, et al. Genetic alterations in goblet cell carcinoids of the vermiform appendix and comparison with gastrointestinal carcinoid tumors. Mod Pathol 2003;16:1189-1198.

28 Ramnani DM, Wistuba II, Behrens C, et al. K-ras and p53 mutations in the pathogenesis of classical and goblet cell carcinoids of the appendix. Cancer 1999;86:14-21.

29 Zauber P, Berman E, Marotta S, et al. Ki-ras gene mutations are invariably present in low-grade mucinous tumors of the vermiform appendix. Scand J Gastroenterol 2011;46:869-874.

30 Yantiss RK, Panczykowski A, Misdraji J, et al. A comprehensive study of nondysplastic and dysplastic serrated polyps of the vermiform appendix. Am J Surg Pathol 2007;31:1742-1753. 